https://doi.org/10.46344/JBINO.2021.v010i01.29

\title{
ROLE OF PHARMACISTS IN THE MANAGEMENT AND PREVENTION OF PNEUMONIA IN ADULTS
}

\section{Senthilraja.M*}

Professor, Department of Pharmaceutical Chemistry J.K.K.Nattraja College of Pharmacy Komarapalayam $638183 \quad$ Namakkal DT, Tamilnadu, India

Email: rajdanish2k@gmail.com

\begin{abstract}
Pneumonia is a lung infection that is causing mild to severe illness across all ages and the common causative organisms include viruses, bacteria and fungi. An individual at high risk of experiencing pneumonia including adults in the age group of 65 years or older, the children's younger than 5 years and those who are having poor or underlying health conditions and smokers'. Common causes of viral pneumonia are influenza and respiratory syncytial virus. Bacterial pneumonia is commonly caused by streptococcus pneumoniae (pneumococcus) and it is important for patients to get the pneumococcal vaccine for prevention.
\end{abstract}

Keywords: Pneumonia; Pharmacists; Treatment; Community; Hospital; 


\section{Community-Obtained Pneumonia (COP)}

Treatment recommendations pharmacist can ensure that patients receive appropriate treatment for communityobtained pneumonia (COP), which is received outside of the hospital setting. The COP treatment guidelines were recently updated to recommend amoxicillin as a first-line agent unless patients have a penicillin allergy ${ }^{2}$. Healthy outpatient adults with COP who do not risk factors for antibiotic resistant pathogens should receive one of the following treatments:

amoxicillin $1 \mathrm{~g}$ 3times a day (first line) or doxycycline $100 \mathrm{mg}$ twice a day or a macrolides (azithromycin $500 \mathrm{mg}$ on the first day then $250 \mathrm{mg}$ daily or clarithromycin $500 \mathrm{mg}$ twice a day or clarithromycin extended release $1 \mathrm{~g}$ daily) ${ }^{3}$. Evidence suggests that pneumococcal resistance (over 30\%) can be associated with macrolides so this should be taken into consideration when selecting COP treatments ${ }^{3}$ Pharmacists should need to educate patients about cardiac risks associated with azithromycin, including QTC prolongation and assess for any potential drug interactions $^{3}$. Patients with communityobtained pneumonia and comorbidities should receive broad spectrum antibiotic treatment in the outpatient setting ${ }^{3}$. Combination therapy with amoxicillin / clavulanate or a cephalosporin (cefpodoxime, cefuroxime) and a macrolide (azithromycin, clarithromycin) or doxycycline should be given as initial treatment3. The reserve fluoroquinolone monotherapy (levofloxacin, moxifloxacin, or gemifloxacin) can be given for patients with drug-resistant COP or B-lactam allergies ${ }^{3}$.Educate patients about serious adverse effects (AEs) that have been associated with fluoroquinolones including tendinitis, tendon rupture, hypoglycemia, peripheral neuropathy and mental health issues ${ }^{4}$.

Individuals who have hospitalized with community-obtained pneumonia will typically require a combination therapy with a B-lactam (ampicillin plus sulbactam, or cefotaxime, ceftriaxone, or ceftaroline) and a macrolide ${ }^{3}$. Fluoroquinolone monotherapy (levofloxacin or moxifloxacin) can also be used to these patients. Patients with contraindications to macrolides and fluoroquinolones can be advised to give combination therapy with a B-lactam and doxycycline.

\section{Hospital-Attained Pneumonia (HAP)}

Hospital-Attained Pneumonia (HAP) and Ventilator-Associated Pneumonia (VAP) Nosocomial pneumonia may occur during a hospital stay and patients may experience ventilator-associated pneumonia (VAP) after being on a ventilator $^{5}$. If conditions are severe, patients can be sending to the intensive care unit (ICU) with pneumonia due to any causative organisms including bacteria, 
virus and fungi. Patients admitted to critical care units are also more likely to have complicating comorbidities, baseline immunosuppression, or other risk factors for multidrug-resistant organisms. For example mechanical ventilation, indwelling venous catheters, parenteral nutrition, renal replacement therapy or nursing home residence. "The pharmacists' roles in these cases are essential as they can ensure that patients receive ventilator pneumonia precaution order sets and that certain medications are being replaced regularly to prevent bacterial colonization and administration lines for propofol and lipids. Pharmacists can play an integral role in proper antimicrobial prophylaxis against expected opportunistic infections for immune compromised patients. If a patient has been previously infected by a multidrug-resistant organism or had a recent antimicrobial exposure within the past Ninety days, an infection prophylaxis and treatment regimens must be modified to ensure that the resistance patterns are being adequately covered and to minimize the likelihood of developing an additional resistance ${ }^{5}$.

\section{COVID-19-Based Pneumonia (CBP)}

Patients with COVID-19 pneumonia and those who are critically ill with nonCOVID-19 pneumonias are having a similar condition and in a similar fashion, as these both conditions are considered as infectious part and an acute respiratory failure component to the illnesses. More information is surfacing to suggest that recovery from COVID pneumonia is only the beginning, as ICU survivors of COVID pneumonia may still need to battle residual symptoms and a slowing

down to return to baseline functioning upon their discharge.

\section{Vaccine Recommendations}

The Food and Drug Administration issued an Emergency Use Authorization for remdesivir for the treatment of hospitalized patients with COVID-19.The National Institutes of Health $^{6}$ (NIH) treatment guidelines were recently updated recommending prioritizing limited supplies of remdesivir for hospitalized patients with COVID-19 who require supplemental oxygen but who are not on high-flow oxygen, non- invasive ventilation, mechanical ventilation, or extracorporeal membrane oxygenation (ECMO), as there is an inadequate evidence of clinical benefit for these individuals ${ }^{7}$.The $\mathrm{NIH}$ guidelines were also recently updated to recommend the corticosteroid such as dexamethasone based on the preliminary results obtained from the randomized evaluation of COVID-19 therapy trial ${ }^{7,8}$. Preliminary data revealed that the mortality rate was lower among patients who were randomized to receive dexamethasone than among those who received standard of care ${ }^{8}$. Based on these results, the $\mathrm{NIH}$ recommends using dexamethasone $6 \mathrm{mg}$ per day for up to 10 days for the treatment of COVID-19 to the patients who aremechanically 
ventilated and in those individuals who require supplemental oxygen but who arenot mechanically ventilated. Clinicians should not use dexamethasone for the treatment of

COVID-19 in patients who do not require supplemental oxygen. The dexamethasone access has become challenging because many wholesalers have run out of the drug. Clinicians may use alternative corticosteroids such as prednisone, methylprednisolone, or hydrocortisone if dexamethasone is not available.

\section{Conclusion}

\section{Tactics for Pneumonia Prevention}

The main role of pharmacists is they should educate patients about the pneumococcal vaccine and its administration to the recommended population given in table nol. As individuals with influenza can also have a chance to develop pneumonia, it is important for pharmacists to encourage patients to get an annual flu vaccine to prevent this complication. Patients get a lowering the risk of pneumonia by ensuring that chronic medical conditions are controlled and also by quitting smoking habits. Additionally, a frequent hand washing with soap and water, wearing a mask and social distancing are effective ways to prevent COVID19 and complications including pneumonia. 
TableNo.1. Adult Pneumococcal Vaccine Administration Recommendations

\begin{tabular}{|c|c|}
\hline NO & Vaccine Administration Recom \\
\hline \multicolumn{2}{|r|}{$\begin{array}{l}\text { Pharmacists should consult CDC's PneumoRecs VaxAdvisor mobile app as a point -of- } \\
\text { care resource to determine which pneumococcal vaccine patients require. }\end{array}$} \\
\hline 1. & $\begin{array}{l}\text { Adults aged 19-64 years(Not previously vaccinated) } \\
\text { Patients with cerebrospinal fluid leaks or cochlear should receive } 1 \text { dose of } \\
\text { pneumococcal conjugate vaccine (pcv13 or prevnar13) followed by a dose of } \\
\text { pneumococcal polysaccharide vaccine (ppsv23 or pneuovax23) at least } 8 \text { weeks } \\
\text { later. }\end{array}$ \\
\hline 2. & $\begin{array}{l}\text { Immunocompromized Adults aged 19-64 years(Not previously vaccinated) } \\
\text { Conditions: Sickle cell disease or hemoglobinopathies, asplenia, } \\
\text { congenital or acquired immunodeficiency, HIV infection, chronic renal failure, } \\
\text { neprotic syndrome, cancer (leukemia,lymphoma,multiple } \\
\text { myloma, Hodgkin disease, iatrogenic immunosuppression } \\
\text { (immunosuppressive drugs, radiation therapy, solid organ transplant) } \\
\text { Individuals should receive one dose of PCV13 first, followed by the first } \\
\text { PPSV23dose at least } 8 \text { weeks later. The secondPPSV23 dose should be given at } \\
\text { least } 5 \text { years after the first PPSV23 dose. }\end{array}$ \\
\hline 3. & $\begin{array}{l}\text { Adults aged 19-64 years with the following chronic conditions (Not previously } \\
\text { vaccinated) should receive one dose of PPSV23. } \\
\text { - Alcoholism } \\
\text { - Chronic Heart Disease - Chronic Liver Disease - Chronic Lung Disease } \\
\text { - Diabetes ( COPD, asthma, emphysema) - Complications from cigarette } \\
\text { smoking }\end{array}$ \\
\hline & $\begin{array}{l}\text { Adults } 65 \text { years and older without an immunocompromising conditions, } \\
\text { cochlear implant, cerebrospinal fluid. } \\
\text { - Receive } 1 \text { dose of PPSV } 23 \\
\text { Updated recommendation to engage in shared clinical decision making } \\
\text { regarding PCV13(For individuals who have not previously received the vaccine) } \\
\text { Patients who received any doses of PPSV } 23 \text { before age } 65 \text { should have } 1 \text { final } \\
\text { dose at } 65 \text { years or older. } \\
\text { The last dose should be administered at least } 5 \text { years after the prior PPSV } 23 \text { dose. }\end{array}$ \\
\hline
\end{tabular}




\section{References:}

1. Metlay JP, Waterer GW, Long AC, et al. Diagnosis and treatment of adults with community-acquired pneumonia: an official clinical practice guideline of the American Thoracic Society and Infectious Diseases Society of America. Am J Respir Crit Care Med. 200 (7), 2019, 45-67.

2. FDA reinforces safety information about serious low blood sugar levels and mental health side effects with fluoroquinolone antibiotics; requires label changes.

FDA.

https://www.fda.gov/drugs/drug-safetyand-availability/fda-reinforces-safety information-about-serious-low-blood-sugarlevels-and-mental-health-side

3. Kalil AC, Metersky ML, Klompas M, Management of adults with hospitalacquired and ventilator-associated pneumonia: Clinical Practice guidelines by the Infectious Diseases Society of America and the American Thoracic Society. Clin Infect Dis. 63(5), 2016, 61- 111.

4. Horby P, Lim WS, Emberson JR, Collaborative Group. Dexamethasone in hospitalized patients with COVID-19preliminary report. N. Engl J Med.17, 2020, 256-275.

5. Remdesivir EUA Letter of Authorization FDA,2000.https://www.fda.gov/media/137 564
6. Covis-19 treatment guidelines of $\mathrm{NIH}$, 2020.

7. Pneumococcal vaccination: Summary of who and when to vaccinate. CDC. 21, 2019.

https://www.cdc.gov/vaccines/vpd/pneu mo/hcp/who-when-to-vaccinate.html.

8. Causes of pneumonia, CDC 9, 2020. https://www.cdc.gov/pneumonia/causes. html

9. Pneumonia can be prevented vaccines can help: CDC 21, 2020. 